Supporting Information for

\title{
Template Regeneration in Galvanic Replacement: A Route to Highly Diverse Hollow Nanostructures
}

Zhuangqiang Gao, ${ }^{\dagger}$ Haihang Ye, ${ }^{\dagger}$ Qingxiao Wang, ${ }^{\S}$ Moon J. Kim, ${ }^{\S}$ Dianyong Tang, ${ }^{\downarrow}$ Zheng Xi, ${ }^{\dagger}$ Zhiyuan Wei, ${ }^{\dagger}$ Shikuan Shao, ${ }^{\dagger}$ and Xiaohu Xia ${ }^{\dagger, t, *}$

†Department of Chemistry, University of Central Florida, Orlando, Florida 32816, United States

${ }^{\ddagger}$ NanoScience Technology Center, University of Central Florida, Orlando, Florida 32816,

United States

${ }^{\S}$ Department of Materials Science and Engineering, University of Texas at Dallas, Richardson, Texas 75080, United States

International Academy of Targeted Therapeutics and Innovation, Chongqing University of Arts and Sciences, Chongqing 402160, People's Republic of China

*Corresponding author. E-mail:Xiaohu.Xia@ucf.edu 


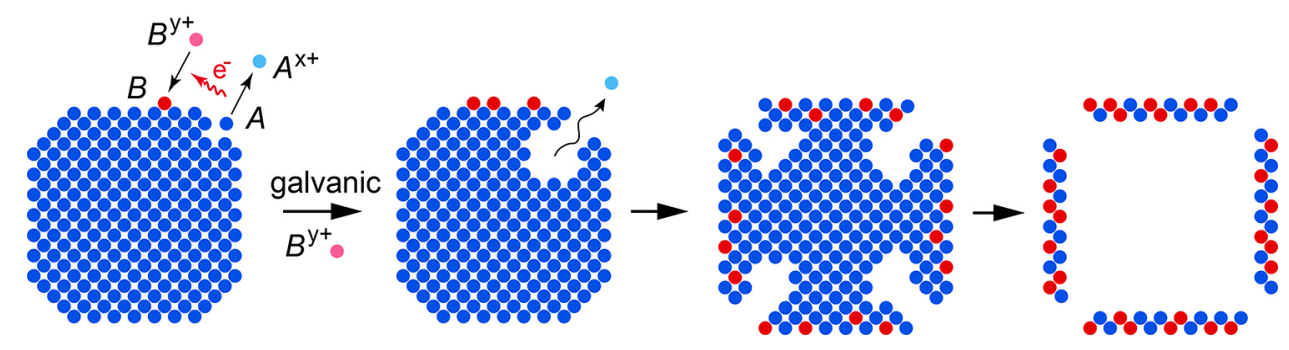

Figure S1. Schematics showing the galvanic replacement between a cubic template of metal $A$ with ions of metal $B\left(B^{y^{+}}\right)$that generates $A-B$ alloyed nanocage. When a cubic template of metal $A$ is in contact with $B^{\mathrm{y}+}$ ions having a higher reduction potential, galvanic replacement reaction $-y / x A_{(\mathrm{s})}+B^{y^{+}}{ }_{(\mathrm{aq})} \rightarrow y / x A^{x^{+}}{ }_{(\mathrm{aq})}+B_{(\mathrm{s})}-$ is initiated at the site with the highest surface energy (in this case, corners). Consequently, atoms of metal $A$ are oxidized and dissolved into the solution in the form of $A^{x^{+}}$ions, generating small holes on the surface of the template. Meanwhile, the electrons released from metal $A$ will be captured by $B^{\mathrm{y}^{+}}$to produce atoms of metal $B$ through a reduction reaction. The newly formed atoms of metal $B$ tend to deposit on the surface of the template. The small holes will serve as the primary sites for continuous dissolution of metal $A$. As more atoms of metal $B$ are deposited on the surface, $A-B$ alloy is formed. Eventually, complete dissolution of metal $A$ transforms the template into a nanocage. More details can be found in the literature. ${ }^{\mathrm{S} 1, \mathrm{~S} 2}$ 


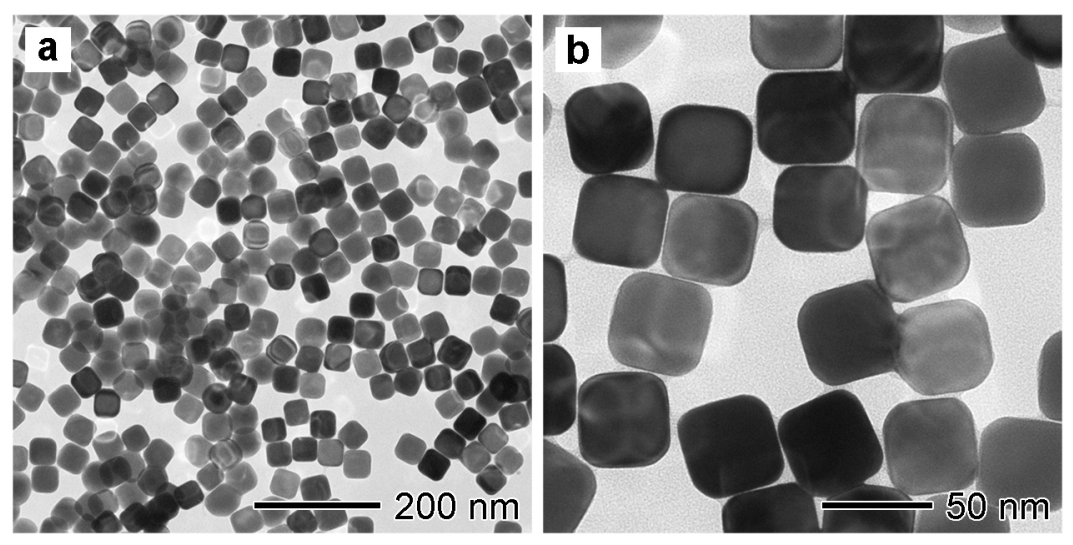

Figure S2. TEM images of Ag nanocubes. Low- (a) and high- (b) magnification TEM images of PVP-capped Ag nanocubes with an average edge length of $42.5 \mathrm{~nm}$. 


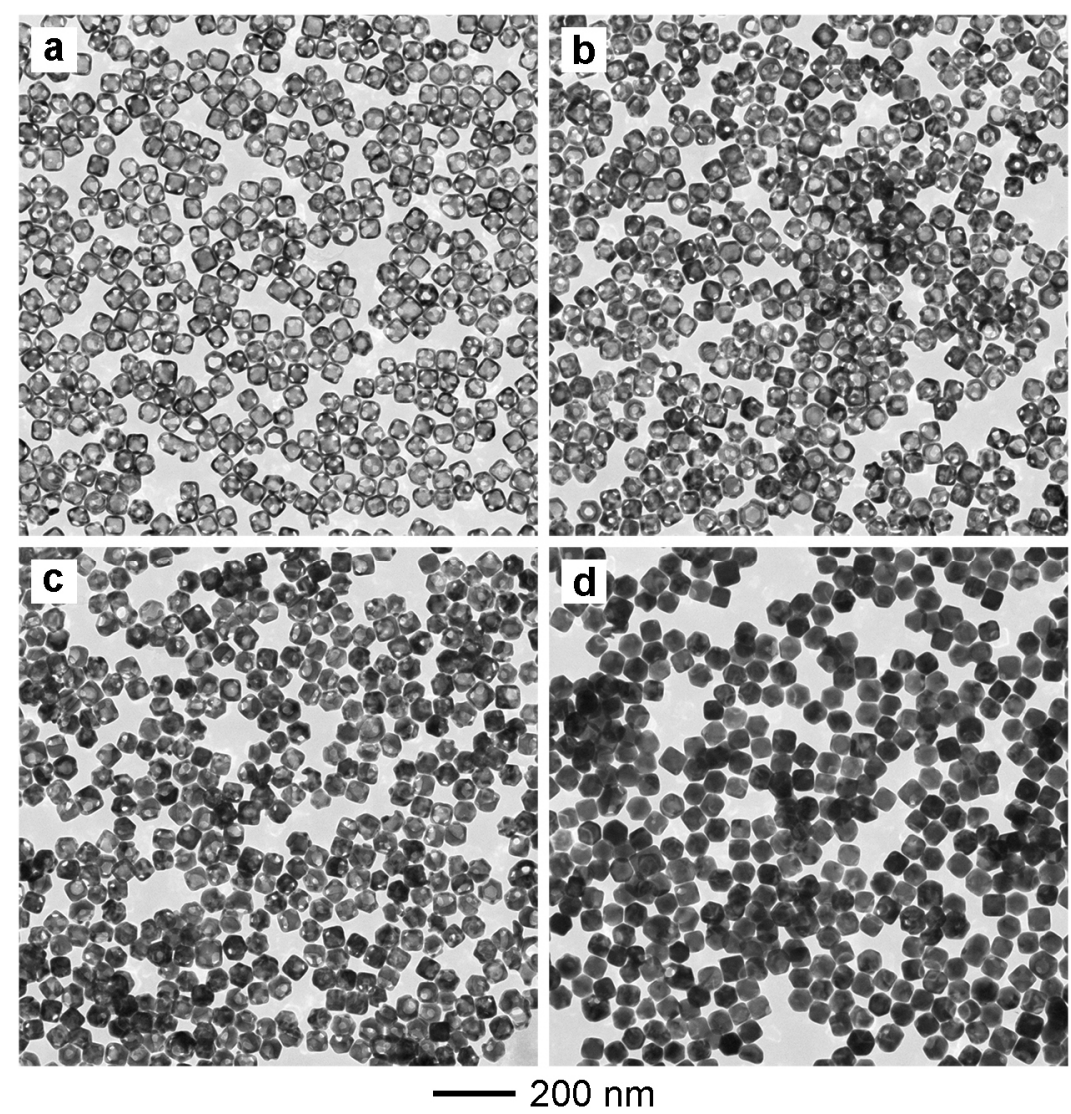

Figure S3. Additional TEM images relative to the TEM images shown in Figure 2a-d. Lowmagnification TEM images of the Ag-Au nanostructures shown in Figure 2a-d, respectively. The $200 \mathrm{~nm}$ scale bar applies to all images. 


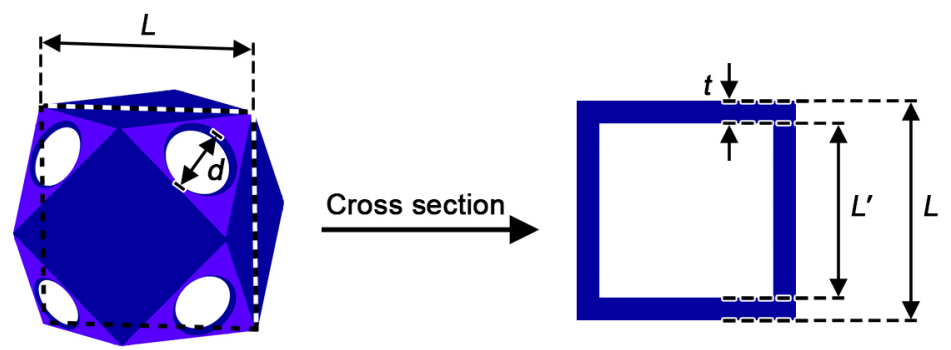

\begin{tabular}{|c|c|c|c|c|}
\hline & $L^{\prime}(\mathrm{nm})$ & $L(\mathrm{~nm})$ & wall thickness $(t, \mathrm{~nm})$ & pore size $(d, \mathrm{~nm})$ \\
\hline$[\mathrm{Ag}-\mathrm{Au}]_{1}$ cage, sample in Fig. $2 \mathrm{a}$ & 41.4 & 48.0 & 3.3 & 15.9 \\
\hline$[\mathrm{Ag}-\mathrm{Au}]_{1.5}$ cage, sample in Fig. $\mathrm{S} 6$ & 41.4 & 51.8 & 5.2 & 14.0 \\
\hline$[\mathrm{Ag}-\mathrm{Au}]_{2}$ cage, sample in Fig. $3 a$ & 41.4 & 55.8 & 7.2 & 11.9 \\
\hline$[\mathrm{Ag}-\mathrm{Au}]_{3}$ cage, sample in Fig. $3 \mathrm{~b}$ & 41.4 & 62.6 & 10.6 & 8.0 \\
\hline$[\mathrm{Ag}-\mathrm{Au}]_{4}$ cage, sample in Fig. $3 \mathrm{c}$ & 41.4 & 69.2 & 13.9 & 4.2 \\
\hline$[\mathrm{Ag}-\mathrm{Au}]_{5}$ cage, sample in Fig. $3 d$ & 41.4 & 76.6 & 17.6 & 0 \\
\hline
\end{tabular}

Figure S4. Physical parameters of $[\mathbf{A g}-\mathbf{A u}]_{n}$ nanocages. Schematics along with a table showing the physical parameters of $[\mathrm{Ag}-\mathrm{Au}]_{n}$ nanocages in this study. Note, the FDTD simulation results in Figures $6 \mathrm{c}, \mathrm{d}$, and $7 \mathrm{~d}$,e were obtained on this basis of these parameters. 

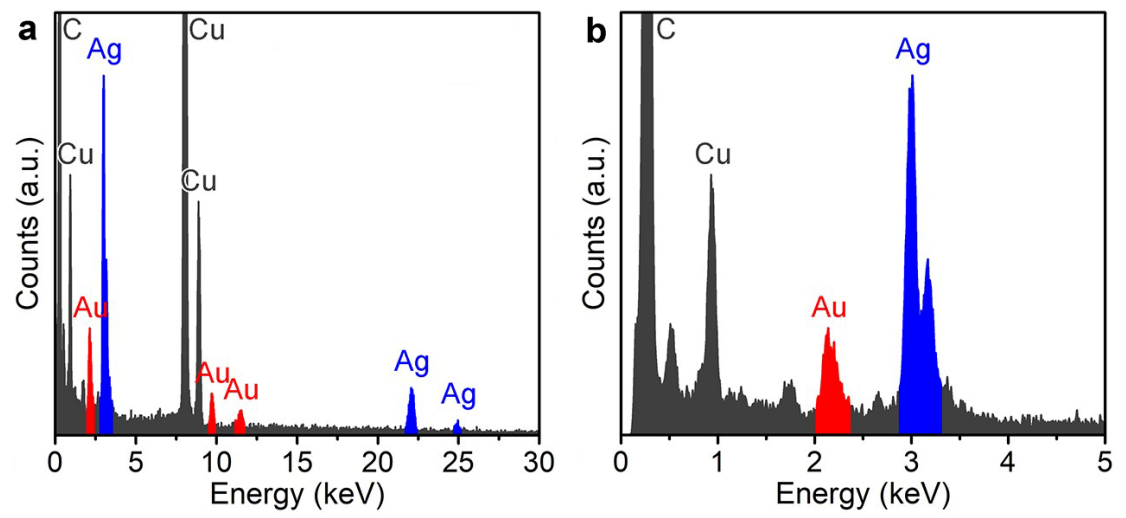

Figure S5. EDX spectra of the Ag@Ag-Au nanocubes (sample in Figure 2d). (a) Full spectra in the energy range of 0-30 keV and (b) magnified EDX spectra in the energy range of 0-5 keV. Peaks of $\mathrm{Ag}$ and $\mathrm{Au}$ are highlighted in blue and red, respectively. Note the signal of $\mathrm{Cu}$ and $\mathrm{C}$ elements were originated from the TEM grids. 

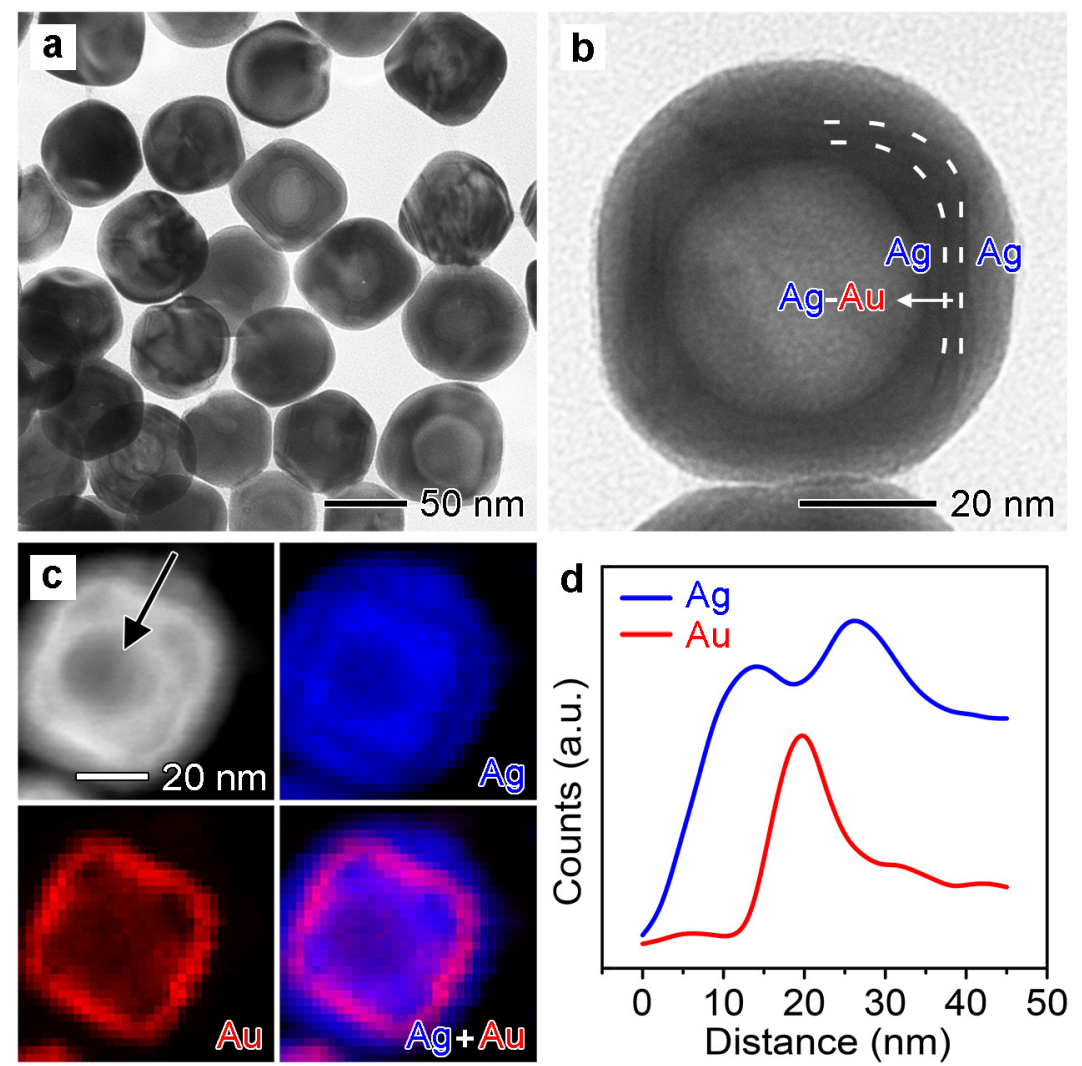

Figure S6. Ag-Au nanostructures obtained by growing Ag on Ag-Au nanocages. a,b, Low(a) and high- (b) magnification TEM images of Ag-Au nanostructures that were obtained by growing Ag on Ag-Au nanocages using the same procedure for preparing Ag@Ag-Au nanocubes shown in Figure $2 \mathrm{~d}$ except that the injection rate for $\mathrm{AgNO}_{3}$ and $\mathrm{AA}$ solution was increased from $5 \mu \mathrm{L} / \mathrm{min}$ to $15 \mathrm{~mL} / \mathrm{min}$ and the concentration of AA solution was increased from $2.5 \mathrm{mM}$ to 250 mM. The difference in contrast between Ag and Ag-Au alloy suggests Ag was grown on both the inner and outer surfaces of Ag-Au nanocages. c,d, EDX mapping images (c) and line-scan EDX spectra (d) of an individual particle, confirming the elemental distributions of $\mathrm{Ag}$ and $\mathrm{Au}$ as marked in $\mathbf{b}$. 


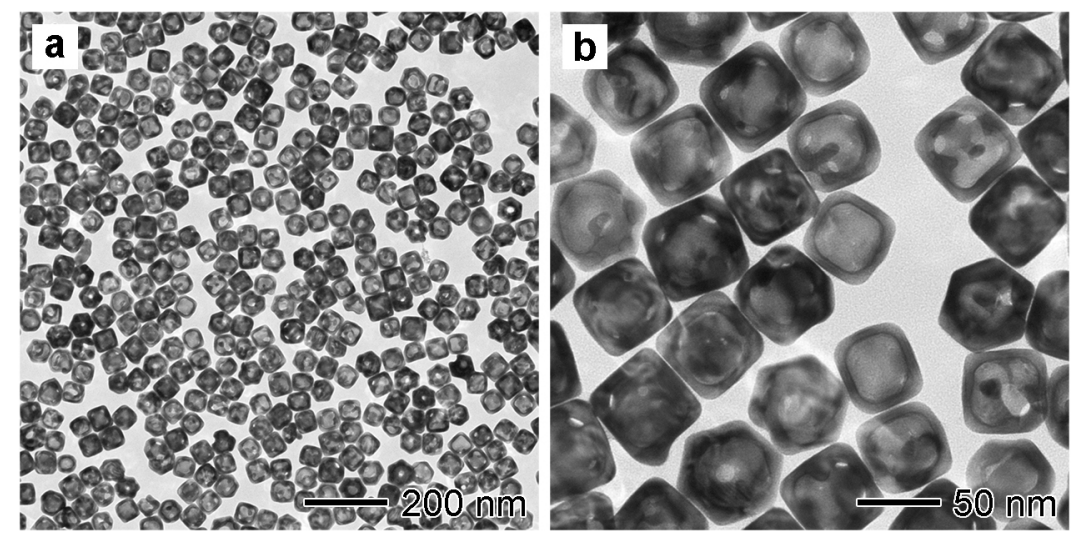

Figure S7. TEM images of $[\mathbf{A g - A u}]_{1.5}$ nanocages. Low- (a) and high- (b) magnification TEM images of the $[\mathrm{Ag}-\mathrm{Au}]_{1.5}$ nanocages that were prepared from the galvanic replacement between the sample in Figure 2c as templates and $\mathrm{Au}^{3+}$. The average wall thickness of these cages was measured to be $5.2 \mathrm{~nm}$. 


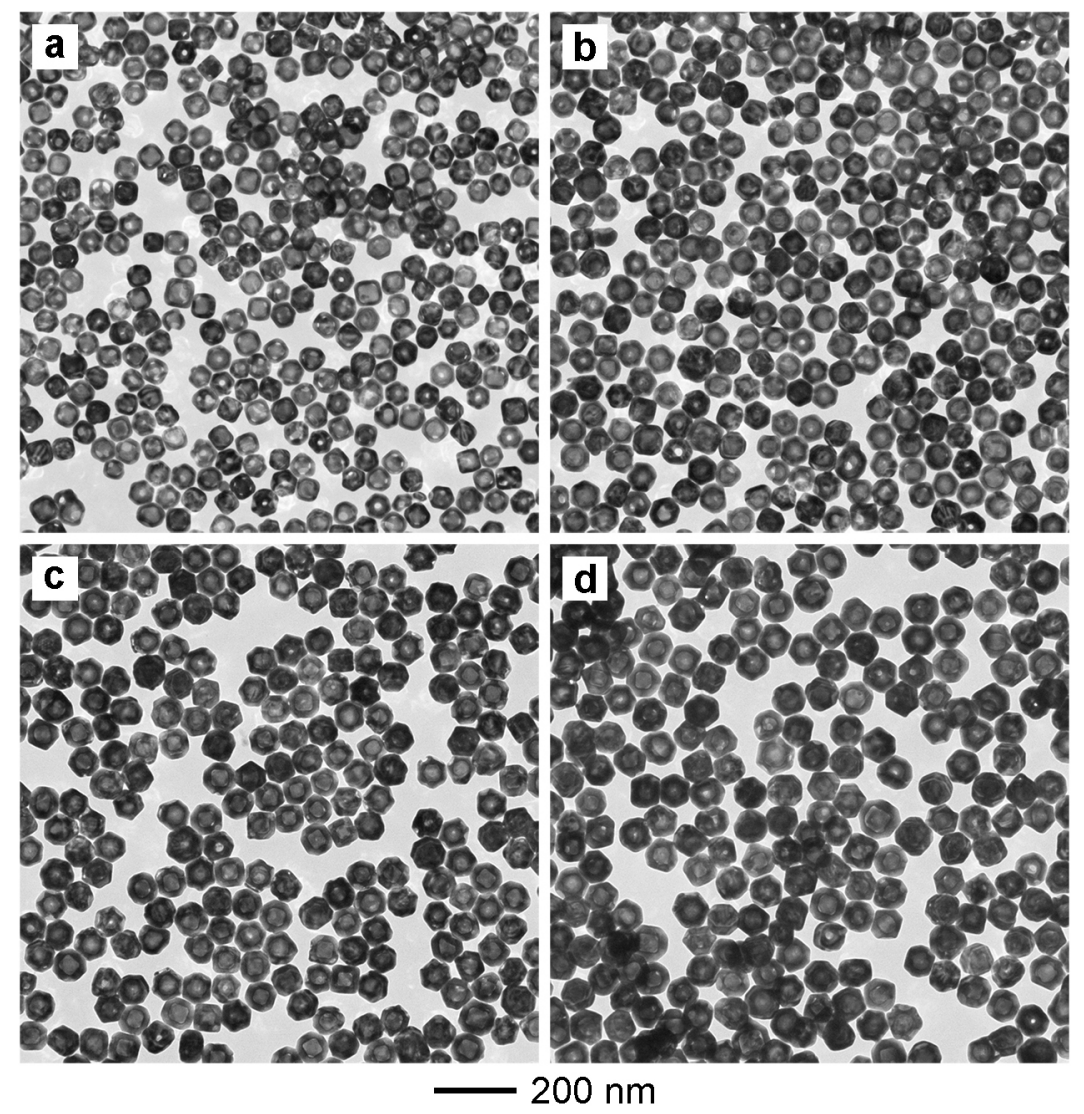

Figure S8. Additional TEM images relative to the images shown in Figure 3a-d. TEM images of $[\mathrm{Ag}-\mathrm{Au}]_{2}(\mathbf{a}),[\mathrm{Ag}-\mathrm{Au}]_{3}(\mathbf{b}),[\mathrm{Ag}-\mathrm{Au}]_{4}(\mathbf{c})$ and $[\mathrm{Ag}-\mathrm{Au}]_{5}(\mathbf{d})$ nanocages at a lower magnification relative to the TEM images shown in Figure 3a-d, respectively. The $200 \mathrm{~nm}$ scale bar applies to all images. 

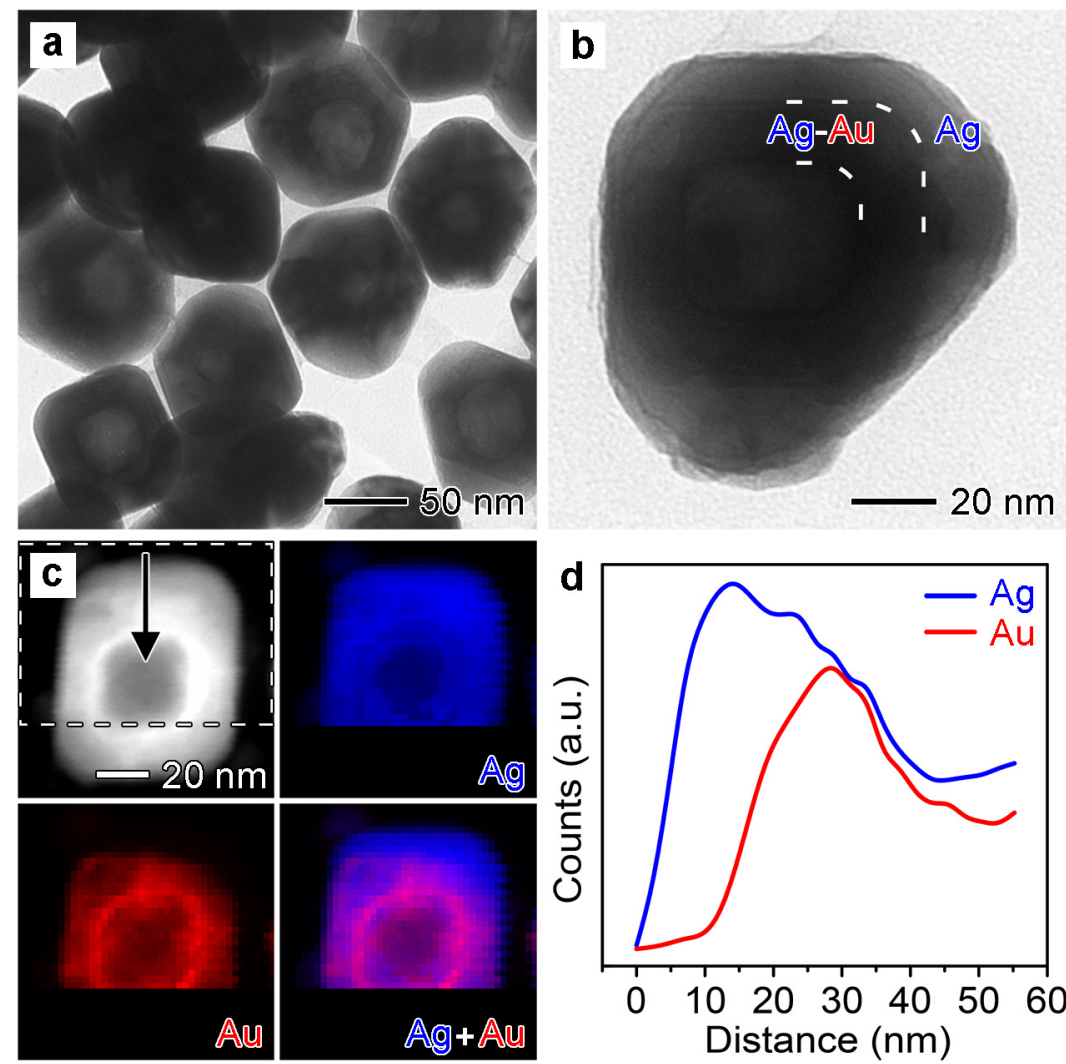

Figure S9. Ag-Au nanostructures obtained by growing Ag on [Ag-Au] 5 cages. a,b, Low- (a) and high- (b) magnification TEM images of nanostructures that were obtained by growing Ag on [Ag-Au] 5 cages (sample in Figure 3d) using the standard procedure of Ag template regeneration. The hollow interiors and exterior Ag layers (with a brighter contrast) of the sample suggest Ag was mainly grown on the outer surfaces of $[\mathrm{Ag}-\mathrm{Au}]_{5}$ cages. c,d, EDX mapping images (c) and line-scan EDX spectra (d) of an individual particle, confirming the elemental distributions of Ag and $\mathrm{Au}$ as marked in $\mathbf{b}$. 


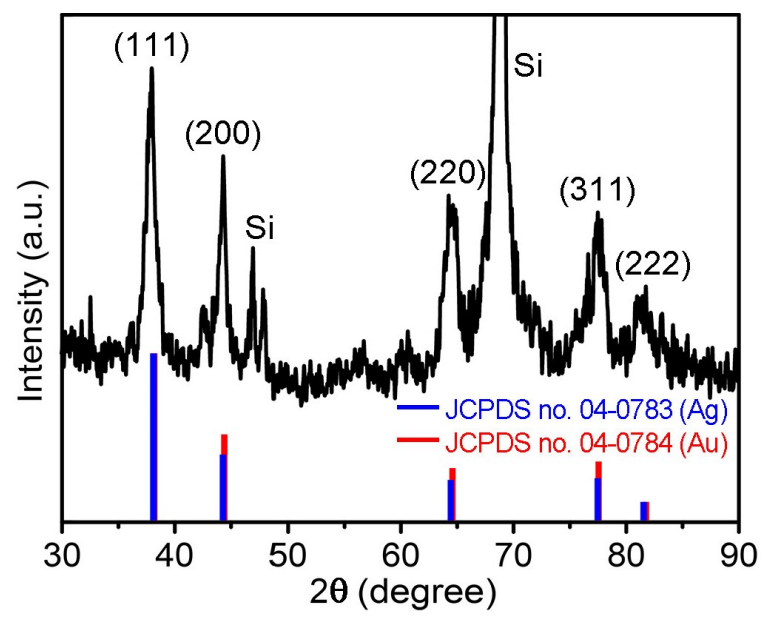

Figure S10. XRD patterns recorded from the $[\mathrm{Ag}-\mathrm{Au}]_{5}$ cages shown in Figure $3 \mathrm{~d}$. 


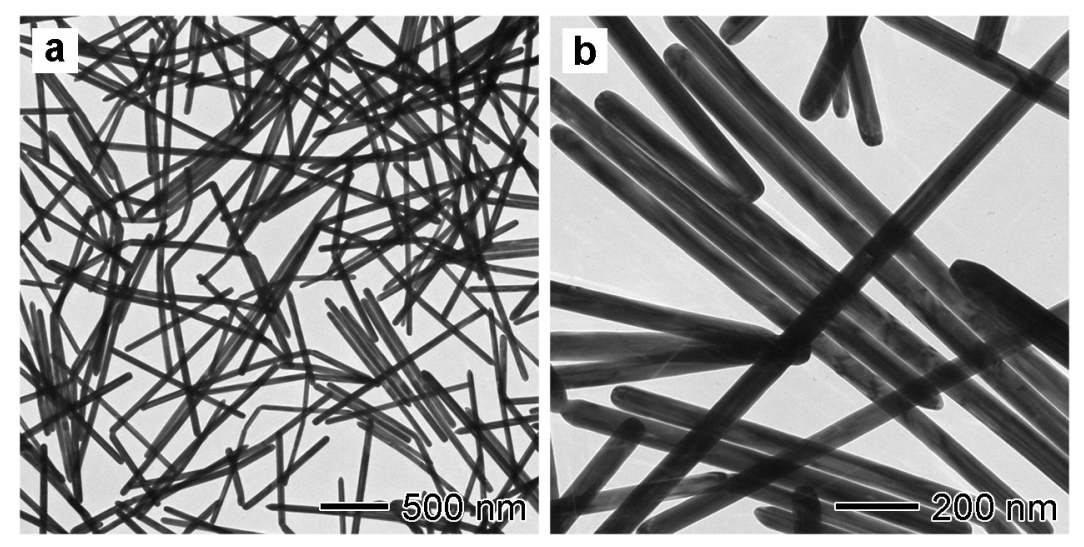

Figure S11. TEM images of Ag nanowires. Low- (a) and high- (b) magnification TEM images of Ag nanowires with an average diameter of $68.0 \mathrm{~nm}$ and length of $2.7 \mu \mathrm{m}$. 

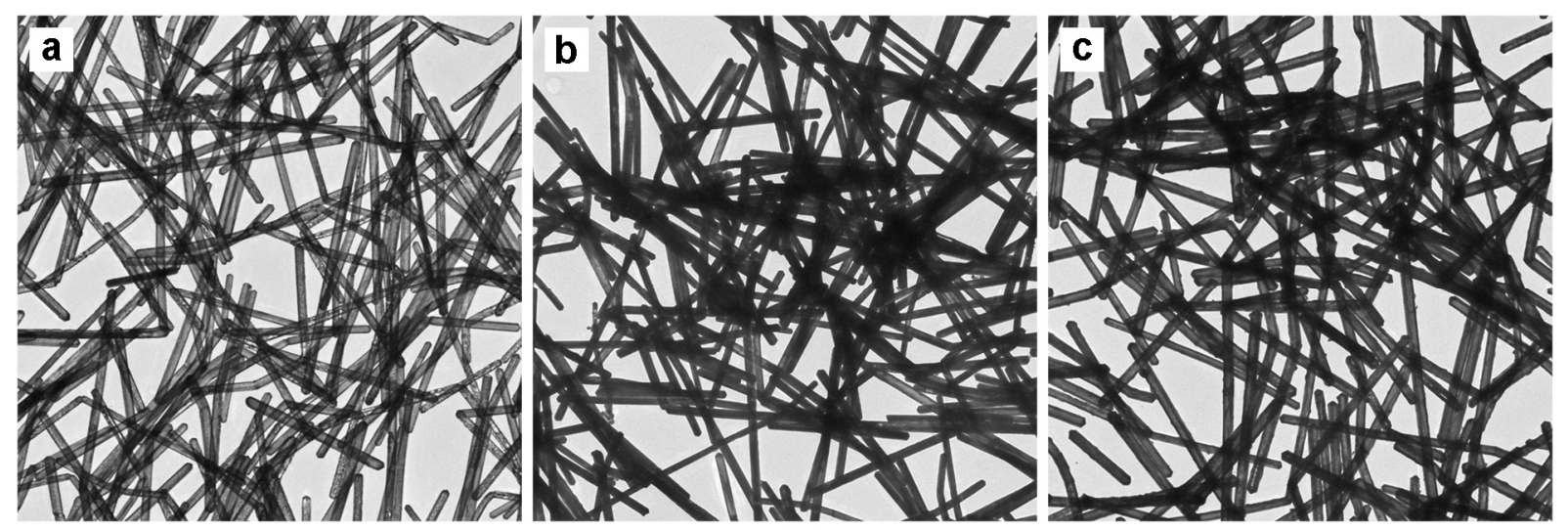

$500 \mathrm{~nm}$

Figure S12. Additional TEM images relative to the images shown in Figure 4a-c. TEM images of Ag-Au nanotubes (a), Ag@Ag-Au core@shell nanowires (b) and $[\mathrm{Ag}-\mathrm{Au}]_{2}$ nanotubes (c) at a lower magnification relative to the TEM images shown in Figure 4a-c, respectively. The $500 \mathrm{~nm}$ scale bar applies to all images. 

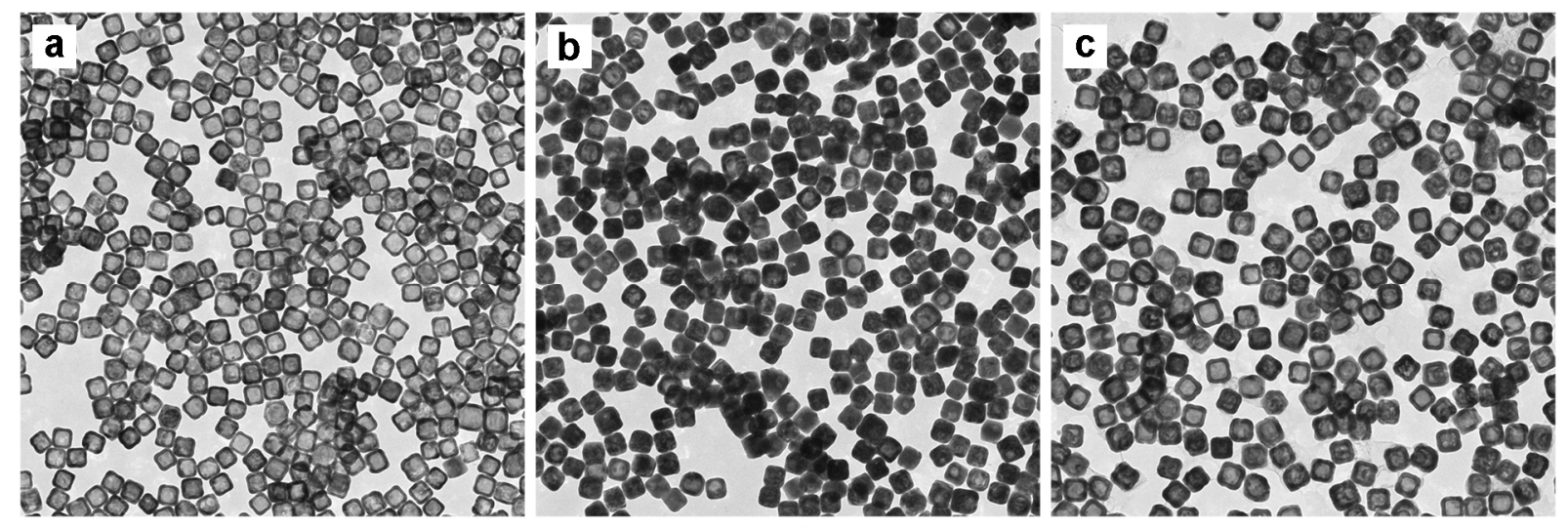

$200 \mathrm{~nm}$

Figure S13. Additional TEM images relative to the images shown in Figure 4f-h. TEM images of Ag-Pd nanocages (a), Ag@Ag-Pd nanocubes (b) and [Ag-Pd] 2 nanocages (c) at a lower magnification relative to the TEM images shown in Fig. 4d-f, respectively. The $200 \mathrm{~nm}$ scale bar applies to all images. 

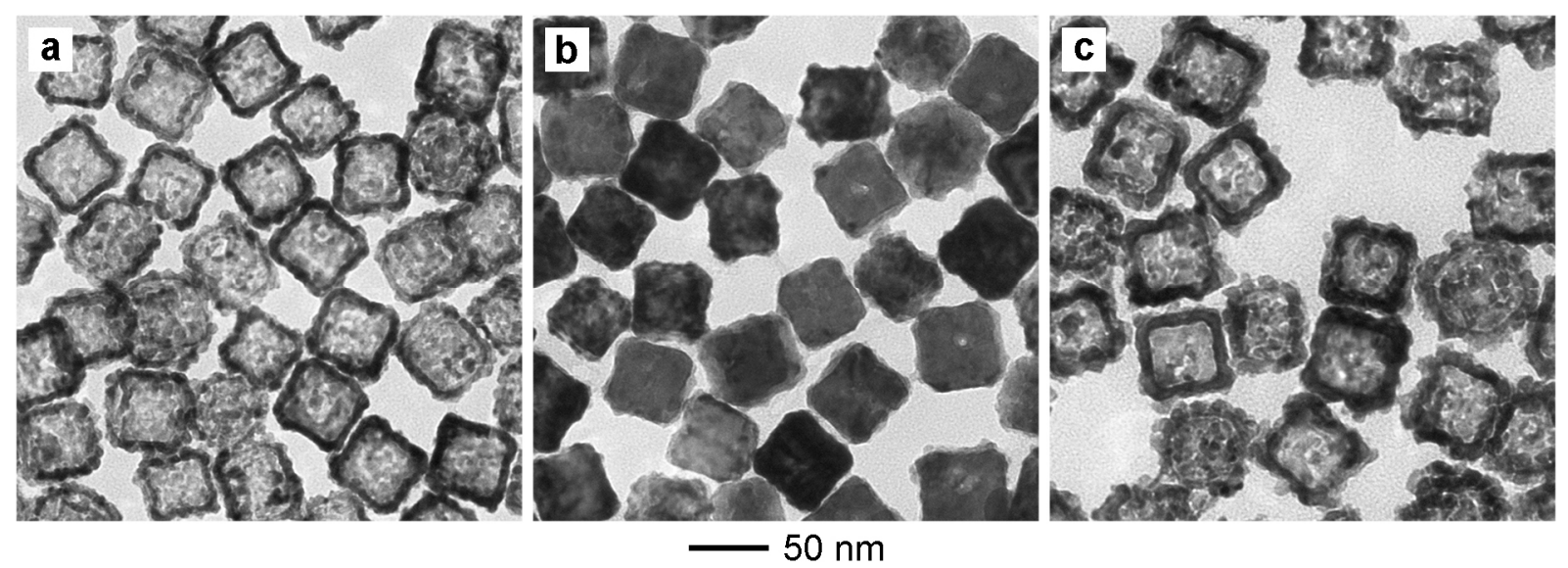

Figure S14. Extension to Ag-Pt bimetallic system. TEM images of Ag-Pt nanocages (a), $\mathrm{Ag} @ \mathrm{Ag}-\mathrm{Pt}$ nanocubes $(\mathbf{b})$, and $[\mathrm{Ag}-\mathrm{Pt}]_{2}$ nanocages $(\mathbf{c})$. 

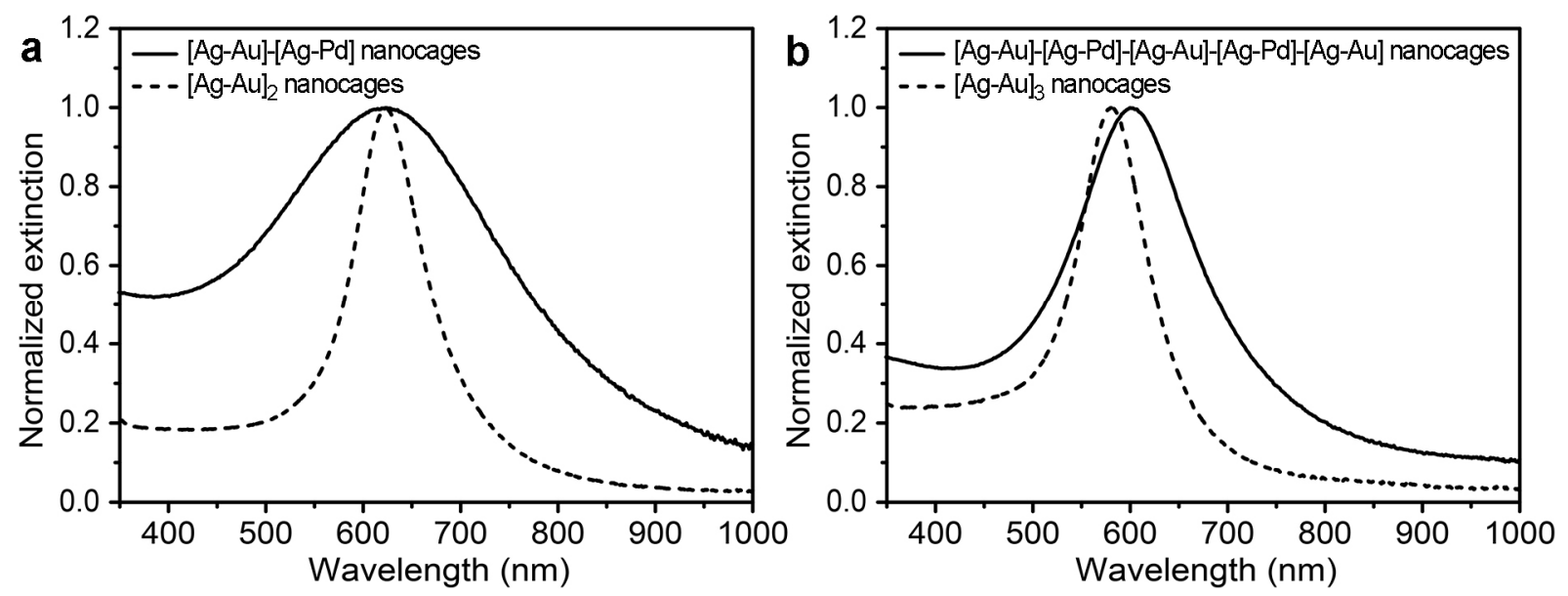

Figure S15. Plasmonic properties of multi-metallic nanocages made of Ag, Au, and Pd. Normalized UV-vis spectra (solid curves) recorded from: (a) [Ag-Au]-[Ag-Pd] nanocages (sample in Figure 5a) and (b) [Ag-Au]-[Ag-Pd]-[Ag-Au]-[Ag-Pd]-[Ag-Au] nanocages (sample in Figure 5e). Dashed curves show the normalized UV-vis spectra recorded from $[\mathrm{Ag}-\mathrm{Au}]_{2}$ and $[\mathrm{Ag}-\mathrm{Au}]_{3}$ nanocages (samples in Figure 3a and 3b, respectively). 


\section{References}

S1. Xia, X.; Wang, Y.; Ruditskiy, A.; Xia, Y. 25th Anniversary Article: Galvanic Replacement: A Simple and Versatile Route to Hollow Nanostructures with Tunable and Well-Controlled Properties. Adv. Mater. 2013, 25, 6313-6333.

S2. Chen, J.; McLellan, J. M.; Siekkinen, A.; Xiong, Y.; Li, Z.-Y.; Xia, Y. Facile Synthesis of Gold-Silver Nanocages with Controllable Pores on the Surface. J. Am. Chem. Soc. 2006, $128,14776-14777$. 